\title{
A novel three-dimensional tumorsphere culture system for the efficient and low-cost enrichment of cancer stem cells with natural polymers
}

\author{
ZHEN-WEI ZHU ${ }^{1,2^{*}}$, LIN CHEN ${ }^{3 *}$, JING-XIAN LIU ${ }^{1}$, JUN-WEN HUANG ${ }^{1}$, \\ GANG WU ${ }^{4}$, YAN-FANG ZHENG $^{4}$ and KAI-TAI YAO ${ }^{1,2}$ \\ ${ }^{1}$ Cancer Research Institute, School of Basic Medical Sciences, Southern Medical University, Guangzhou, Guangdong 510515; \\ ${ }^{2}$ Department of Oncology, Shenzhen Hospital of Southern Medical University, Shenzhen, Guangdong 518000; \\ ${ }^{3}$ Department of Pathology, The First Affiliated Hospital, Sun Yat-Sen University, Guangzhou, Guangdong 510080; \\ ${ }^{4}$ Department of Oncology, Zhujiang Hospital, Southern Medical University, Guangzhou, Guangdong 510280, P.R. China
}

Received April 16, 2017; Accepted August 1, 2017

DOI: $10.3892 /$ etm.2017.5419

\begin{abstract}
Cancer stem cells (CSCs) are considered to serve a key role in tumor progression, recurrence and metastasis. Tumorsphere culture is the most important method for enriching CSCs and is widely used in basic research and drug screening. However, the traditional suspension cell culture system has several disadvantages, including low efficiency, high cost and difficult procedure, making it difficult to produce tumorspheres on a large scale. In the present study, two biomaterials, methylcellulose (MC) and gellan gum (GG), were used to construct a novel culture system based on the traditional system. Subsequently, the characteristics of the novel three-dimensional (3D) culture system were evaluated, the design scheme was optimized, and the morphological and biological features of the tumorspheres cultured in this $3 \mathrm{D}$ system were compared with the traditional system. The results revealed that the tumorspheres cultured in the novel 3D system presented a higher seeding density and improved morphology, while maintaining stem-like properties. This evidence suggests that a simple, efficient and low-cost culture system that produces tumorspheres on a large scale was successfully
\end{abstract}

Correspondence to: Dr Kai-Tai Yao, Cancer Research Institute, School of Basic Medical Sciences, Southern Medical University, 1023-1063 Shatai South Road, Guangzhou, Guangdong 510515, P.R. China

E-mail: ktyao1931@163.com

*Contributed equally

Abbreviations: CSCs, cancer stem cells; 3D, three-dimensional; MC, methylcellulose; GG, gellan gum; qPCR, quantitative polymerase chain reaction

Key words: cancer stem cells, tumorsphere, three-dimensional culture, large-scale, methylcellulose, gellan gum constructed, which can be widely used in various aspects of stem cell research.

\section{Introduction}

Cancer stem cells (CSCs) have been identified as rare cell populations in numerous types of cancer and are considered to be the reason for cancer recurrence, metastasis and therapy resistance in different studies $(1,2)$. The CSC theory provides great opportunities for the fields of cancer research, clinical therapy and drug screening. However, the development of drug screening and theory studies for utilizing CSCs is hindered by the difficulties in isolating and amplifying these cells in vitro due to the low proportion of CSCs. Isolated cells that form tumorspheres are generally recognized as CSCs with self-renewal and tumorigenic capacities $(3,4)$. Thus, the sphere formation assay is widely used for the enrichment and characterization of CSCs in vitro (5). The traditional method involves the culture of tumorspheres using a non-adhesive culture system with a serum-free medium containing growth factors.

However, numerous challenges remain with the traditional suspension culture system, including high cost, low yield and difficult procedure. Certain of the major limitations also include the uncontrollable spontaneous fusion among spheres and adherent sphere growth (6). Therefore, a simple, efficient and low-cost culture system is urgently required. In recent years, various types of materials have been used as three-dimensional (3D) matrices, such as chitosan-alginate scaffolds, collagen, alginate and agarose (7-10). These biomaterials are nontoxic and can alter the rheology properties of the culture system (11).

In the present study, a novel 3D culture system with two natural polymers, methylcellulose (MC) and gellan gum (GG), was established. MC is an excellent thickening agent and stabilizer that is widely used in various types of food, drug delivery systems and cosmetic products (12). In addition, GG is able to form stable network structures in hydrogel solutions (13) and is widely used as a thickening agent, emulsifier and stabilizer (14). The novel culture system described in the 
present study solves several key problems associated with the tumorsphere culture process, including the spontaneous fusion, adherent growth, low yield and difficult procedure. This 3D matrix sphere culture system also provides a useful method for simulating the microenvironment of CSCs in vitro.

\section{Materials and methods}

Tumorsphere culture in the traditional suspension system. The human nasopharyngeal carcinoma (NPC) cell line 5-8F and Sune1, human colon cancer cell line SW480 and lung cancer cell line H460 were obtained from the Cancer Research Institute of Southern Medical University (Guangzhou, China). In addition, the human NPC cell line C666-1 (EBV+) was obtained from Professor George Sai Wah Tsao (The University of Hong Kong, Hong Kong, China). The cell lines used in the current study were cultured in RPMI-1640 medium (Biological Industries, Kibbutz Beit-Haemek, Israel) supplemented with $10 \%$ fetal bovine serum (Biological Industries, Kibbutz Beit-Haemek, Israel) and incubated at $37^{\circ} \mathrm{C}$ with $5 \%$ $\mathrm{CO}_{2}$ for $48 \mathrm{~h}$. The cells were collected, washed to remove serum, and then suspended in serum-free Dulbecco's modified Eagle's medium (DMEM)/F12 (Biological Industries) supplemented with $20 \mathrm{ng} / \mathrm{ml}$ epidermal growth factor, $10 \mathrm{ng} / \mathrm{ml}$ basic fibroblast growth factor and 2\% B27 (1:50 dilution). These cell factors were purchased from PeproTech, Inc. (Rocky Hill, NJ, USA). The cells were cultured in ultra-low attachment 6-well or 96-well plates (Corning, Inc., Corning, NY, USA) at a density of up to 2,000 cells $/ \mathrm{ml}$ and maintained at $37^{\circ} \mathrm{C}$ under $5 \% \mathrm{CO}_{2}$ in a humidified incubator for 7 days.

Novel 3D tumorsphere culture system with natural polymers. The novel 3D tumorsphere culture medium was prepared as follows: MC (R\&D Systems, Inc., Minneapolis, MN, USA) and GG (Sigma-Aldrich; Merck KGaA, Darmstadt, Germany) were mixed together with pure water to a concentration of $3 \%$ (w/v) and dissolved by stirring at room temperature. The fully dissolved solution was sterilized at $160^{\circ} \mathrm{C}$ for $2 \mathrm{~h}$ in an autoclave to remove any endotoxins. Next, the MC and GG solution was added to the serum-free DMEM/F12 supplemented with the various cell factors. Subsequently, the cells (5-8F, Sune1, H460, SW480 and C666-1) were seeded in a culture bottle containing the new culture medium and maintained at $37^{\circ} \mathrm{C}$ under $5 \% \mathrm{CO}_{2}$ in a humidified incubator for 7 days.

Flow cytometry. The expression of the molecular marker CD133 in the various cell cultures was detected using an anti-CD133-phycoerythrin antibody (Miltenyi Biotec $\mathrm{GmbH}$, Bergisch Gladbach, Germany, catalog no. 130-080-801, 1:10). The adherent cells and tumorspheres were harvested, gently disaggregated to single-cell suspensions by trypsin, and stained with CD133-PE for $30 \mathrm{~min}$ in the dark at $4^{\circ} \mathrm{C}$. The stained cells were then detected using a flow cytometer. Data analysis was performed using the FlowJo software 7.6 (FlowJo LLC, Ashland, OR, USA).

Reverse transcription-quantitative polymerase chain reaction $(R T-q P C R)$. After $24 \mathrm{~h}$ for adherent cells and 7 days for tumosphere cells, total RNA was extracted from the cells using an RNAiso Plus reagent (Takara Bio, Inc., Otsu,
Japan). Concentration of RNA was determined by ultraviolet spectrophotometer (ThermoNanoDrop 2000, Thermo Fisher Scientific, Inc., Waltham, MA, USA). RT was then performed using a PrimeScript RT reagent kit (Takara Bio, Inc.), according to the manufacturer's protocol. Subsequently, the SYBR Green RT-qPCR Master Mix (Takara Bio, Inc.) was used to conduct qPCR. The primer sequences used for octamer-binding transcription factor 4 (Oct-4), (sex determining region Y)-box 2 (Sox2), Nanog and GAPDH are reported in Table I. GAPDH was used as the reference gene. The PCR reactions involved an initial denaturation at $95^{\circ} \mathrm{C}$ for $2 \mathrm{~min}$, followed by 42 cycles at $95^{\circ} \mathrm{C}$ for $15 \mathrm{sec}, 60^{\circ} \mathrm{C}$ for $30 \mathrm{sec}$, and then a final incubation at $72^{\circ} \mathrm{C}$ for $30 \mathrm{sec}$. Data from the RT-qPCR experiments were analyzed according to the $2^{-\Delta \Delta \mathrm{Cq}}$ method (15).

Western blot analysis. Cells were lysed using a Protein Extraction kit (P0027; Beyotime Institute of Biotechnology, Beijing, China). Whole cell protein concentrations were measured using BCA reagent (P0010S; Beyotime Institute of Biotechnology). Lysates ( $25 \mu \mathrm{g}$ total protein) were separated by $12 \%$ SDS-PAGE and transferred to polyvinylidene difluoride membranes. The membranes were incubated in blocking buffer (3\% bovine serum albumin; Sigma-Aldrich; Merck KGaA) at room temperature for $1 \mathrm{~h}$. Subsequently, the membranes were incubated at $4^{\circ} \mathrm{C}$ overnight with the following primary antibodies: GAPDH (ab9485; 1:2,500), Oct-4 (ab19857; 1:1,000), Nanog (ab109250; 1:1,000) and Sox2 (ab97959; 1:1,000; all purchased from Abcam, Cambridge, MA, USA). Goat anti-rabbit secondary antibody conjugated with peroxidase (BS13278; 1:5,000; Bioworld Technology, Inc., St Louis Park, MN, USA) was used as the secondary detection antibody at $25^{\circ} \mathrm{C}$ for $1 \mathrm{~h}$. The immunoblots were then detected using an enhanced chemiluminescence reagent (Pierce; Thermo Fisher Scientific, Inc.) on the ChemiDoc XRS+ system (Bio-Rad Laboratories, Inc., Hercules, CA, USA). Images were analyzed using Image Lab software 3.0 (Bio-Rad Laboratories, Inc.).

Immunofluorescence analysis. The tumorspheres were centrifuged at $200 \mathrm{x} \mathrm{g}$ for $5 \mathrm{~min}$ to remove media, washed with PBS and collected in EP tubes at room temperature. Next, the tumorspheres were fixed in $4 \%$ paraformaldehyde in the EP tubes. Rabbit anti-human primary antibodies, including Sox2 (ab97959; 1:1,000), Oct4 (ab19857; 1:200) and Nanog (ab109250; 1:200; all from Abcam), were added and incubated overnight at $4^{\circ} \mathrm{C}$ using a shaking table. Subsequent to washing the tumorspheres three times with PBS, goat anti-rabbit secondary antibodies conjugated with Cy3 (P0183; Beyotime Institute of Biotechnology; 1:1,500) were added, and the tumorspheres were incubated at room temperature for $1 \mathrm{~h}$. DAPI was used as the nuclear stain (blue). Images were obtained using fluorescence microscopy.

Tumorsphere formation and limiting dilution assays. In order to assess the tumorsphere formation efficiency, single adherent cells were seeded in a series of duplicate wells with different numbers of cells per well in a 96-well plate. The traditional tumorsphere medium or the new 3D tumorsphere medium was added to the 96-well plates, and cells were seeded at 1,5,10 and 20 cells/well. The typical tumorsphere is $>100 \mu \mathrm{m}$ in diameter and round in shape. The wells containing tumorspheres were 
Table I. Forward and reverse sequences of the quantitative polymerase chain reaction primers.

\begin{tabular}{lll}
\hline Primer & \multicolumn{1}{c}{ Forward } & \multicolumn{1}{c}{ Reverse } \\
\hline Sox2 & 5'-GCCGAGTGGAAACTTTTGTCG-3' & 5'-GGCAGCGTGTACTTATCCTTCT-3' \\
Oct4 & 5'-CTGGGTTGATCCTCGGACCT-3' & 5'-CCATCGGAGTTGCTCTCCA-3' \\
Nanog & 5'-TTTGTGGGCCTGAAGAAAACT-3' & 5'-GAAGGTGAAGGTCGGAGTC-3' \\
GAPDH & 5'-AGGGCTGTCCTGAATAAGCAG-3' & 5'-GAAGATGGTGATGGGATTTC-3'
\end{tabular}

Sox2, (sex determining region Y)-box 2; Oct4, octamer-binding transcription factor 4.

counted as positive wells, and those without formation of tumorspheres were considered as negative wells. Subsequently, the frequency of tumorsphere formation was calculated using an extreme limiting dilution algorithm (ELDA software; http://bioinf.wehi.edu.au/software/elda/).

Chemoresistance assays. Adherent cells and sphere-forming cells were seeded at a density of 1,000 cells per well in 96-well culture plates containing different concentrations of cisplatin $(1,2,4$, and $8 \mu \mathrm{g} / \mathrm{ml}$; Sigma-Aldrich; Merck $\mathrm{KGaA}$ ) and medium alone (RPMI-1640 medium serving as the control of adherent cells and tumorspheres). The cell viability was then measured using the cell counting kit- 8 assay (C0037; Beyotime Institute of Biotechnology), according to the manufacturer's protocol.

Xenograft in vivo experiment. Animal experiments were conducted in strict accordance with the recommendations in the Guide for the Care and Use Committee of Southern Medical University and approved by the Experimental Animal Ethics Committee of Southern Medical University. Adherent cells and sphere-forming cells were derived from the 5-8F cell line. Adherent and sphere-forming cells were injected into left and right dorsal thighs of mouse, respectively, at the same dose of cells $\left(1 \times 10^{5}\right.$ cells in the first group, $1 \times 10^{4}$ cells in the second group). The nude BALB/c mouse ( $n=6$; age, 4 weeks; weight, 14-16 g; Laboratory Animal Center of Southern Medical University) were subcutaneously injected with $1 \times 10^{4}$ or $1 \times 10^{5}$ cells resuspended in $100 \mu \mathrm{l}$ serum-free solution mixed with PBS and Matrigel (BD Biosciences, Franklin Lakes, NJ, USA; Matrigel to PBS ratio=1:1) at 4 weeks of age. The animals were housed in micro-isolator cages under pathogen-free conditions, at $20^{\circ} \mathrm{C}$ and $50 \%$ humidity, with a 12-h light/dark cycle, in the animal facility of Southern Medical University. The mice were observed to record tumor size and then euthanized at 4 weeks after the cells were injected.

Statistical analyses. Statistical analyses were performed with the SPSS version 20.0 software package (IBM Corp., Armonk, NY, USA). Unpaired Student's t-test was used to detect significant differences in the mean values when two groups were compared regarding the fusion rate and relative quantitative expression of genes. Comparisons in the stem cell frequencies between any of the groups were estimated by Pearson's $\chi^{2}$ tests. All statistical tests were two-sided. P-values of $<0.05$ were considered to indicate differences that were statistically significant, and are detailed in the figure legends.

\section{Results}

Design of the novel 3D tumorsphere culture system. In order to reduce the aggregate fusion and sedimentation of tumorspheres, the characteristics of several biological materials (agar, matrigel, collagen, MC, GG and xanthan gum) were compared in a preliminary experiment. These polymers were added to the traditional tumorsphere culture medium, and it was observed that MC and GG were able to alter the rheology properties of the medium. Therefore, a 3D tumorsphere culture system was then successfully established using these two polymers (Fig. 1A and B). Improved results were obtained using higher concentrations of MC and GG.

The concentration of MC was optimized in increments in order to balance the sphere fusion and viscosity, and the concentration of $0.3 \%(\mathrm{w} / \mathrm{v}) \mathrm{MC}$ was selected. It was demonstrated that this concentration decreased the average fusion rate from $15.8 \pm 3.9$ to $2.9 \pm 1.0 \%$ for the NPC cell line $5-8 \mathrm{~F}$ $(n=3 ; P=0.005 ;$ Fig. $1 C$. Similar results were obtained with the $\mathrm{H} 460$ cell line $(\mathrm{n}=3 ; \mathrm{P}=0.02$; Fig. $1 \mathrm{C})$, with the average fusion rate decreasing from $19.7 \pm 4.0$ to $3.4 \pm 0.7 \%$. Thus, supplementation with MC was useful for suppressing the spontaneous fusion of tumorspheres in the culture system (Fig. 1D, upper panel), while the tumorspheres clustered into a large mass in the traditional system (Fig. 1D, lower panel).

In order to resolve the issue of tumorsphere sedimentation, GG polymer was also added to the tumorsphere culture system. A series of GG concentrations were examined, and it was determined that $0.03 \%$ was the most appropriate concentration. This concentration of GG was adequate for maintaining the tumorspheres in suspension for at least $48 \mathrm{~h}$ (Fig. 1E). Furthermore, it was observed that the spheres were distributed in a three-dimensional manner in the culture medium (data not shown). Thus, utilizing the properties of MC and GG, a novel 3D culture system was obtained.

Evaluation of the novel 3D tumorsphere culture system. In the present study, a novel 3D culture system with two natural polymers was established. Initially, the permissible highest seeding density was estimated for the new culture system. The highest seeding density of cells reached $10^{5}$ cells $/ \mathrm{ml}$ in the new system, which was nearly 100 times greater in comparison with that in the traditional system $(1,000-2,000$ cells/ml). However, the high seeding density of the cells consumed large volumes of the medium.

Next, the morphological characteristics of the tumorspheres during their growth process in the novel 3D culture system 
A

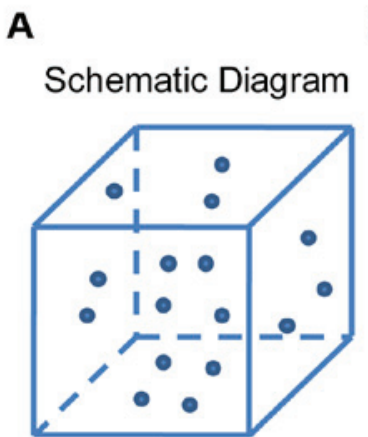

D

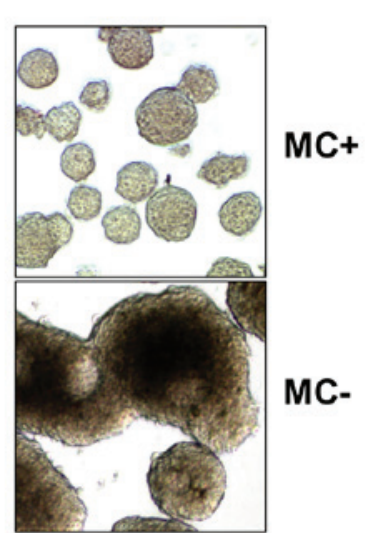

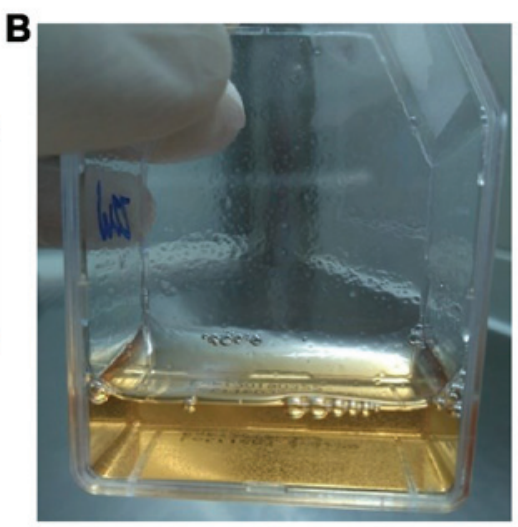

C

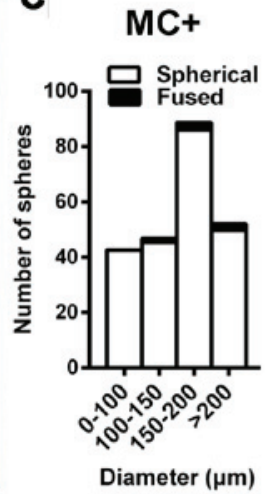

MC-

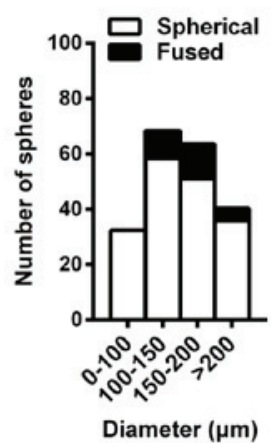

E

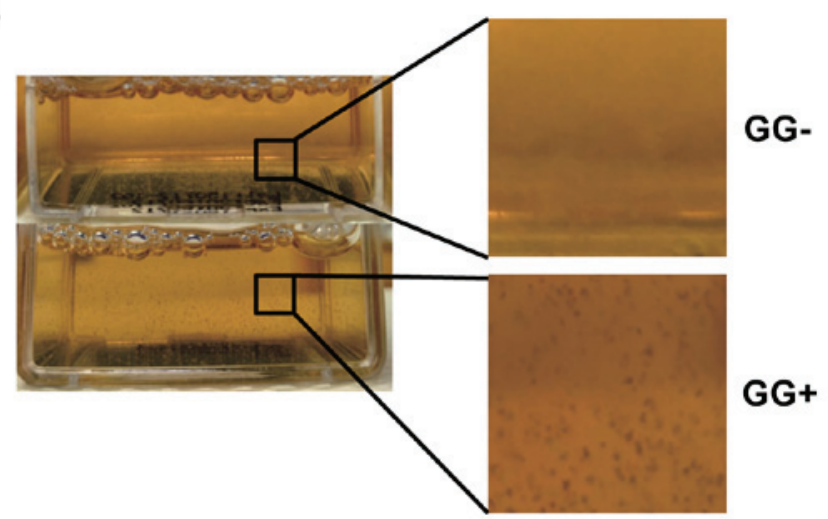

Figure 1. Evaluation of the novel three-dimensional tumorsphere culture system. (A) Schematic diagram of the culture system. (B) A photograph of the new culture system in which tumorspheres were suspended without agitation. (C) Differences in the morphology of the tumorspheres in the culture medium with or without MC. (D) Suspension characteristics of tumorspheres in the culture medium with or without $0.03 \%$ GG. (E) Size distribution of the tumorspheres derived from 5-8F cells with or without MC. MC, MC, methylcellulose; GG, gellan gum.

A Seeding cells

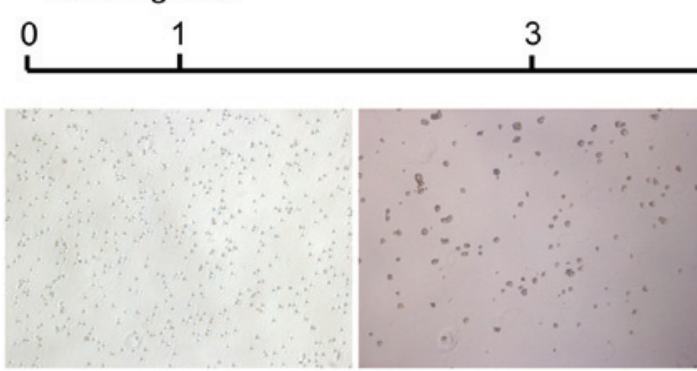

B

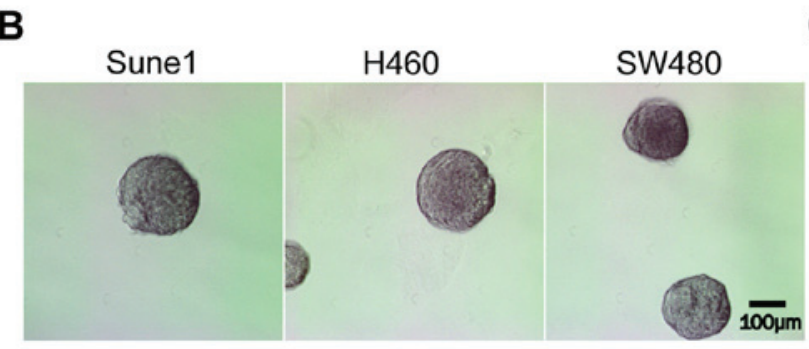

Medium change
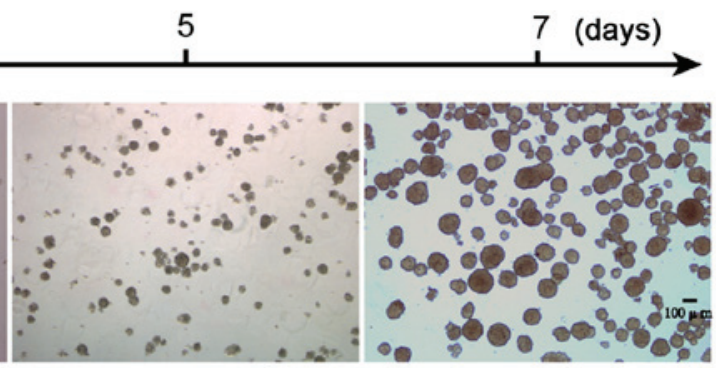

C C666-1

C666-1

\section{Traditional suspend New 3D}
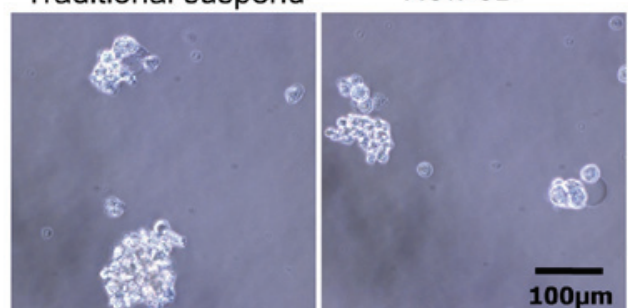

Figure 2. Growth of the tumorspheres in the novel 3D culture system. (A) Morphological characteristics during the growth of tumorspheres for 7 days. (B) Formation of tumorspheres in the Sune1, H460 and SW480 cell lines. (C) The C666-1 cell line did not form tumorspheres in the traditional or 3D culture systems. 3D, three-dimensional.

were evaluated. As shown in Fig. 2A, certain cells underwent apoptosis or cell death during the first 2 days of culture due to the non-adhesive and suspended environment. After $\sim 5$ days, the diameters of the tumorspheres reached 50-100 $\mu \mathrm{m}$ (Fig. 2A). 
A

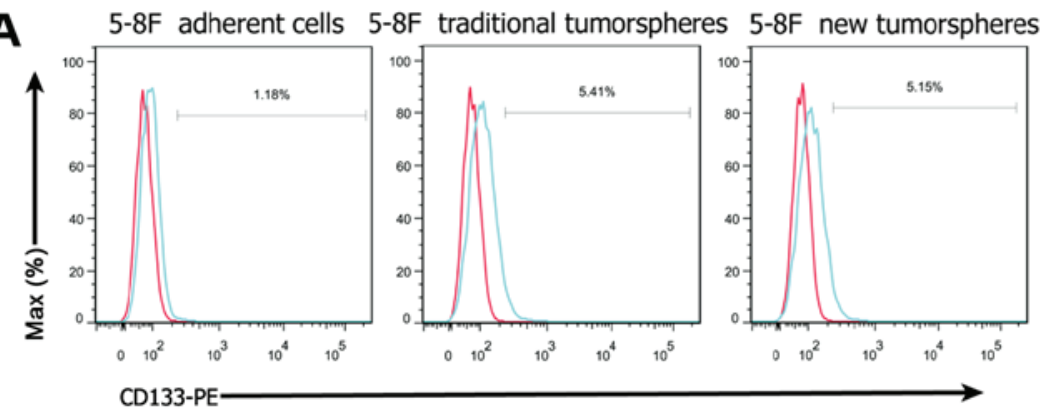

H460 adherent cells $\mathrm{H} 460$ traditional tumorspheres $\mathrm{H} 460$ new tumorspheres
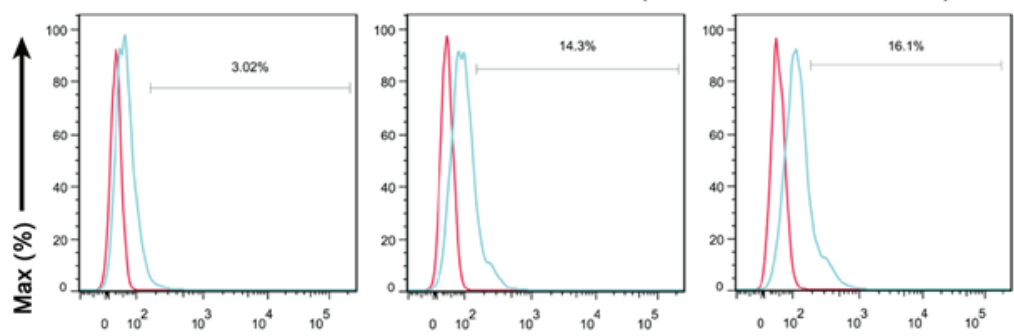

CD133-PE
B

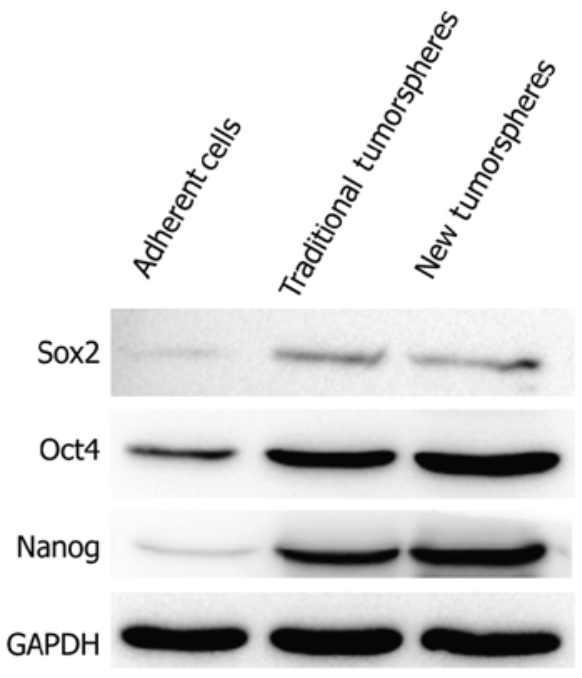

D

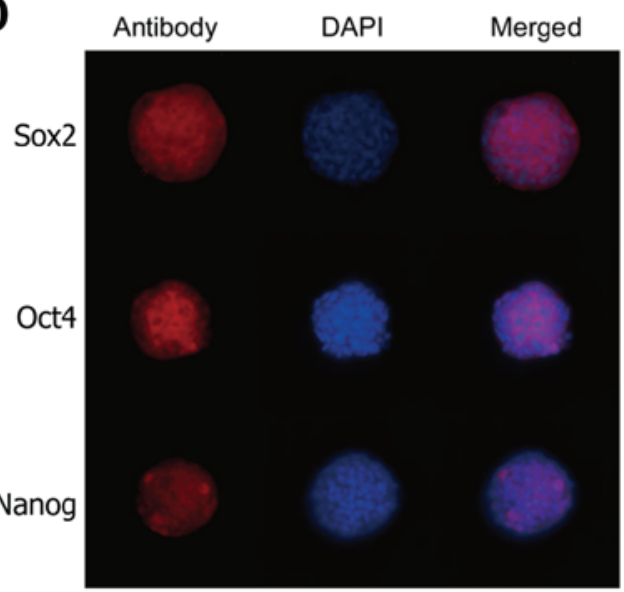

Figure 3. Stemness-associated properties of tumorspheres cultured in the traditional or new 3D system. (A) Flow cytometric analysis of CD133 expression in the adherent cells and sphere-forming cells. (B) Relative transcriptional levels of stemness-associated genes, including Sox 2 , Oct4 and Nanog, in the adherent cells and tumorspheres. (C) Western blot analysis of the protein expression levels stemness-associated genes in 5-8F cells cultured as adherent cells and sphere-forming cells. ${ }^{*} \mathrm{P}<0.05,{ }^{* *} \mathrm{P}<0.01$ and ${ }^{* * *} \mathrm{P}<0.001$ vs. adherent cells. (D) Immunofluorescence staining of Sox 2 , Oct 4 and Nanog in the tumorspheres of the novel $3 \mathrm{D}$ culture system in $5-8 \mathrm{~F}$ cells. 3D, three-dimensional; Sox 2, (sex determining region Y)-box 2; Oct4, octamer-binding transcription factor 4.

After 7 days of culture, the diameters of the tumorspheres reached 100-200 $\mu \mathrm{m}$, and the tumorspheres were similar in size, approximately spherical in shape and smooth in contour (Fig. 2A). Only a small number of tumorspheres underwent spontaneous fusion in the novel culture system.

In addition, this culture system we successfully used to produce tumorspheres in Sune1, H460 and SW480 cell lines (Fig. 2B). However, the C666-1 cell line was unable to form typical tumorspheres in the new and the traditional culture systems (Fig. 2C).

Stemness-associated properties of tumorspheres. The expression of the putative CSC marker CD133 of NPC and lung cancer was then examined. The results revealed that the proportion of sphere-forming cells positively stained in the two culture systems was significantly higher compared with that of the parental adherent cells (Fig. 3A). In the 5-8F and $\mathrm{H} 460$ cell lines, the percentage of sphere-forming cells expressing CD133 was nearly 4-fold higher when compared with that of the parental adherent cells (Table II; Fig. 3A). The expression of CD133 was absent or very low in the parental cells. However, there were no significant differences in the proportion of positive cells between the new culture system and the traditional one.

The study next evaluated the expression of stemness-associated genes at the transcriptional and translational levels. The results revealed that the protein and mRNA expression levels of Sox 2, Oct4 and Nanog were significantly higher in the tumorspheres compared with their parental cells, although there were no significant differences between the tumorspheres cultured in the two systems (Fig. 3B and C). The immunofluorescence staining of the tumorspheres was also assayed 
Table II. CD133-positive cells detected by flow cytometry (mean \pm standard deviation).

\begin{tabular}{lccc}
\hline $\begin{array}{l}\text { Cell } \\
\text { lines }\end{array}$ & $\begin{array}{c}\text { Adherent } \\
\text { cells }\end{array}$ & $\begin{array}{c}\text { Traditional } \\
\text { tumorspheres }\end{array}$ & $\begin{array}{c}\text { New } \\
\text { tumorspheres }\end{array}$ \\
\hline $5-8 \mathrm{~F}$ & $1.15 \pm 0.11$ & $5.16 \pm 0.27$ & $5.16 \pm 0.30$ \\
$\mathrm{H} 460$ & $3.04 \pm 0.27$ & $15.10 \pm 0.92$ & $15.37 \pm 0.64$ \\
\hline
\end{tabular}

(Fig. 3D). The results revealed that the stemness-associated proteins were expressed in tumorspheres.

Analysis of tumorsphere formation efficiency. To obtain accurate data, the limiting dilution method was used (Fig. 4A). The results revealed that there was no significant difference in the tumorsphere formation rate (CSC frequency) between the traditional suspension culture system and the novel 3D culture system (5-8F cell line: Fold change $=0.870, \mathrm{P}=0.544$; H460 cell line: Fold change $=0.877, \mathrm{P}=0.591$; Fig. $4 \mathrm{~A}$ ). In the traditional suspension culture system, the estimated frequency of CSC was $1 / 16.2$, with a confidence interval of $1 / 11.7-22.4$, while this frequency was $1 / 14.1$ with a confidence interval of 1/10.3-19.3 in the novel 3D culture system of the 5-8F cell line (Fig. 4B). Similar results were obtained for the H460 cell line.

Chemoresistance of the tumorspheres. To assess whether the sphere-forming cells cultured in the novel 3D system possessed chemoresistance properties, the study investigated the cell survival rate of $5-8 \mathrm{~F} / \mathrm{H} 460$ tumorspheres and parental cells treated with cisplatin. The survival rates of the sphere-forming cells in the two culture systems were compared with those of the 5-8F adherent cells. Upon treatment with 1,2,4 and $8 \mu \mathrm{g} / \mathrm{ml}$ cisplatin, the cell survival in the traditional system was altered by 1.04-, 1.12-, 1.18- and 1.50-fold, and in the new system was 1.05-, 1.08-, 1.23- and 1.51-fold, respectively. Similar results were obtained for the H460 cell line, with 1.05-, 1.05-, 1.12and 1.80-fold change in the traditional system, and 1.07-, 1.06-, 1.15 - and 1.72-fold in the new system for 1, 2, 4 and $8 \mu \mathrm{g} / \mathrm{ml}$ cisplatin treatment, respectively. In addition, it was noted that the higher drug concentration was accompanied by a higher fold change in the survival rate. The results presented in Fig. 4C revealed that the sphere-forming cells were more resistant to chemotherapy compared with the adherent cells, which may explain why current therapies fail to eradicate CSCs. The fold changes were not significantly different between tumorspheres cultured in the two systems with 5-8F and $\mathrm{H} 460$ cell lines ( $\mathrm{P}=0.770$ and $\mathrm{P}=0.893$, respectively; Fig. $4 \mathrm{C})$.

Xenograft experiments. The results of xenograft experiments indicated that as few as $10^{4}$ sphere-derived $5-8 \mathrm{~F}$ cells were able to develop tumors $(1 / 3$ mice; indicated by the red circle in Fig. 4D) and that $10^{5}$ sphere-derived cells developed tumors at a higher frequency in the animals $(3 / 3$ mice; red circles in Fig. 4D). By contrast, the required number of parental cells for tumor formation in nude mice was as high as $10^{5}(1 / 3$ mice; black circle in Fig. 4D), indicating that the enrichment of tumor-initiating cells in the spheres was at least 10 -fold higher compared with that in the parental cells. Furthermore, the volume of the tumor from the sphere-forming cells was higher in comparison with that in the parental group in the same mouse. These data revealed that the tumorspheres cultured in the novel 3D system had a stronger ability to initiate tumors.

\section{Discussion}

In the present study, a simple, efficient and low-cost 3D tumorsphere culture system was established for enriching CSCs. By adding natural polymers to the culture system, the spontaneous fusion and sedimentation of the tumor spheres were prevented without disturbing their proliferation and stemness-associated properties. The new culture system may offer an attractive alternative method to amplify CSCs in vitro, with potential wide applications in CSC research and drug screening.

Great efforts have been made to develop effective methods to study CSCs, with tumorsphere culture being an excellent such method, since the sphere-forming cells possess stemness-associated properties and the tumorspheres are able to enrich CSCs (16). The tumorsphere culture method has been widely applied to enrich and isolate CSCs via a non-adhesive culture system with a serum-free medium containing defined growth factors. The main mechanisms involved in the formation of tumorspheres under suspension conditions are resistance to apoptosis and activation of stemness-associated pathways (17). Only cells with stem cell-like properties can grow and form spheres under non-adherent conditions. However, the traditional suspension culture system has several disadvantages due to the spontaneous fusion among the spheres and adherent sphere growth during the culture process (6). Uncontrollable fusion of the tumorspheres results in spontaneous differentiation of the CSCs, instability in sphere sizes and shapes, as well as the formation of very large spheres. Due to these disadvantages, traditional methods involve the seeding of a small density of cells (between 1,000 and 2,000 cells/ml), leading to low sphere production. Thus, these disadvantages limit the wide application of the traditional method.

In order to address these issues, a novel 3D tumorsphere culture system was designed in the present study. It was attempted to identify natural polymers that could limit the mobility of the tumorspheres or maintain them in suspension. Several commonly used biological materials with good biocompatibility and nontoxicity were tested, including MC, xanthan gum, GG, Matrigel, collagen, alginate and agarose gels (18). These candidates are able to generate 3D matrix structures or increase the viscosity of the medium. In the current study, it was identified that MC and GG met the requirements. $\mathrm{MC}$ is an excellent thickening agent and stabilizer, and the conventional working concentration (1\%) of MC markedly reduces tumorsphere fusion; however, this leads to difficulties in handling during medium replacement and tumorsphere collection $(19,20)$. Therefore, the concentration of MC was optimized in the present study, and 0.3\% (w/v) MC was identified as the appropriate concentration to control the fusion of tumorspheres. It was confirmed that the tumorspheres could be easily collected via centrifugation at this concentration of MC. Thus, the spontaneous fusion among tumorspheres was prevented, even when cells were seeded at a high density $\left(10^{5} \mathrm{cell} / \mathrm{s} / \mathrm{ml}\right)$. Furthermore, GG is a 
A

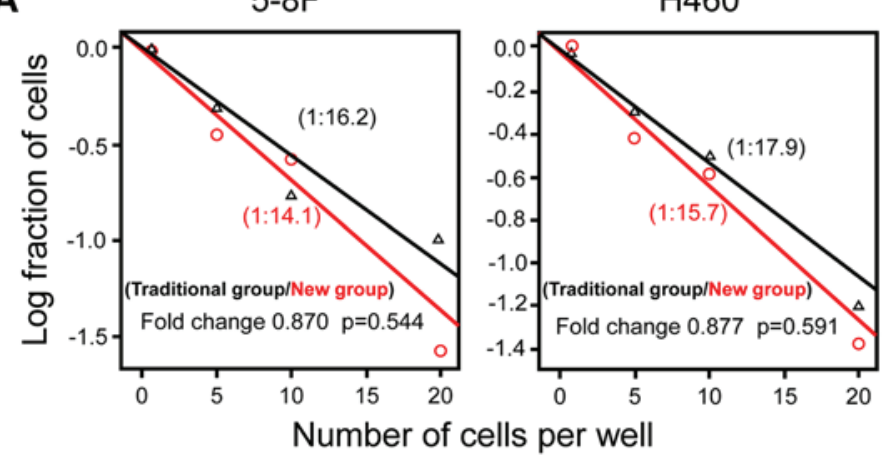

B

Confidence intervals for $1 /$ (stem cell frequency)

$5-8 \mathrm{~F}$

\begin{tabular}{|c|c|c|c|}
\hline Group & Lower & Estimate & Upper \\
\hline Traditional & 22.4 & 16.2 & 11.7 \\
\hline New & 19.3 & 14.1 & 10.3 \\
\hline \multicolumn{4}{|c|}{$\mathrm{H} 460$} \\
\hline Group & Lower & Estimate & Upper \\
\hline Traditional & 25.1 & 17.9 & 12.8 \\
\hline New & 21.8 & 15.7 & 11.4 \\
\hline
\end{tabular}

D

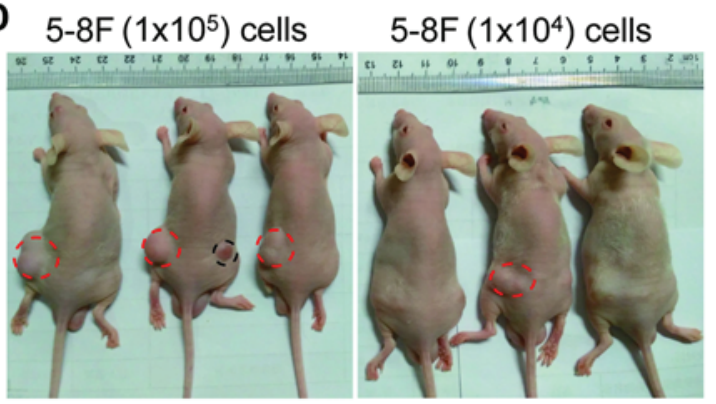

Figure 4. Evaluation of stemness-associated properties of tumorspheres. (A) Tumorsphere formation frequency of 5-8F and $\mathrm{H} 460$ cell lines in the traditional and the novel three-dimensional culture systems. (B) Frequency of cancer stem cells demonstrated no significant differences (5-8F, P=0.544; H460, P=0.591). An extreme limiting dilution algorithm was used to calculate this frequency. (C) Viability was measured using a cell counting kit-8 assay (mean \pm standard deviation; $\mathrm{n}=3$ ). The sphere-forming cells were more resistant to chemotherapy compared with the adherent cells. (D) In vivo tumorigenicity of sphere-forming cells cultured in the novel culture system. Sphere-forming and adherent cells were subcutaneously injected into the left and right thighs of male nude mice, respectively. The volumes of tumors developed from the sphere-forming cells (red circles) were greater compared with those of the adherent cells (black circle) in the same mouse.

water-soluble anionic polysaccharide secreted by bacteria of the genus Sphingomonas (21) and has been demonstrated to form stable network structures in hydrogel solutions (22). GG has been widely used as an immobilization matrix in the food industry and for drug delivery (23). Data in the present study revealed that at very low concentrations $(0.03 \% \mathrm{w} / \mathrm{v}), \mathrm{GG}$ inhibited the sedimentation of tumorspheres and maintained them in suspension without additional devices.

The novel culture system that was established in the present study was a true 3D system, with tumorspheres distributed in a $3 \mathrm{D}$ manner. The study also observed that the majority of the tumorspheres remained suspended in the medium for $>48 \mathrm{~h}$ without agitation. By contrast, in the traditional culture system, the tumorspheres would sink to the bottom of the culture dish due to gravity. In addition, the issues of aggregation and fusion were resolved, which are influenced by various factors, such as vibration of the incubator, shaking of the dish and other minor environmental alterations. In the new culture system, the tumorspheres grew in a relatively close conformation without the interference of adjacent tumorspheres due to the high viscosity of MC. Therefore, the tumorspheres can be cultured at a high seeding density in an ordinary culture flask rather than using low-adhesion dishes, and can be easily amplified on a large scale. Furthermore, numerous tumorspheres are harvested at once, thus reducing the cost and resources required. The results from the experiments with these tumorspheres ensured their reliability and stability for application in future studies to facilitate drug discovery and CSC research.
The morphological and biological features of the tumorspheres cultured in the novel 3D system were then compared with those in the traditional system. The results revealed that the tumorspheres cultured in the novel 3D system had better morphology and a higher seeding density, as well as maintained stemness properties. It was also observed that the tumorspheres cultured in the new system were more similar in size, approximately spherical in shape and smooth in contour, since the MC creates a relatively isolated environment that prevents spontaneous fusion. In the traditional culture system, the fusion of tumorspheres and their irregular shapes hinder their application in CSC research. A notable observation of the present study was that the C666-1 cell line did not form tumorspheres in the two culture systems. This finding suggests that the results obtained in the new system were not false positive. The results clearly demonstrated that the novel 3D culture system is equivalent in function with the traditional one.

Other culture systems established using various biomaterials, such as Matrigel, collagen, alginate and agarose gels, have been established (18). Compared with those culture systems, the culture system reported in the present study used less supplementation, keeping the medium in liquid, thus facilitating the handling of the cultures. Similar to normal stem cells, CSCs are also regulated by the tumor microenvironment $(24,25)$. However, mimicking the microenvironment of CSCs in vitro is challenging due to the lack of the support structure found in tissues. Several studies have stated that the tumorspheres coated with Matrigel closely resembled the 
in vivo conditions (26). Therefore, the microenvironment could be mimicked by adding extracellular matrix components, biomolecules or growth factors into the 3D culture system for future studies $(27,28)$. However, the microenvironment is complex and there is much more work required to improve the novel 3D culture system.

In conclusion, the present study offers a simple, efficient and low-cost 3D tumorsphere culture system with potential wide applications in CSC research and drug screening.

\section{Acknowledgements}

This study was supported by the Sanming Project of Medicine in Shenzhen (Academician Kaitai Yao's Group for Nasopharyngeal Carcinoma Research from Southern Medical University).

\section{References}

1. Flemming A: Cancer stem cells: Targeting the root of cancer relapse. Nat Rev Drug Discov 14: 165, 2015.

2. Adorno-Cruz V, Kibria G, Liu X, Doherty M, Junk DJ, Guan D, Hubert C, Venere M, Mulkearns-Hubert E, Sinyuk M, et al: Cancer stem cells: Targeting the roots of cancer, seeds of metastasis, and sources of therapy resistance. Cancer Res 75: 924-929, 2015.

3. Lim YC, Oh SY, Cha YY, Kim SH, Jin X and Kim H: Cancer stem cell traits in squamospheres derived from primary head and neck squamous cell carcinomas. Oral Oncol 47: 83-91,2011.

4. Fan X, Liu S, Su F, Pan Q and Lin T: Effective enrichment of prostate cancer stem cells from spheres in a suspension culture system. Urol Oncol 30: 314-318, 2012.

5. Weiswald LB, Bellet D and Dangles-Marie V: Spherical cancer models in tumor biology. Neoplasia 17: 1-15, 2015.

6. Zweigerdt R, Olmer R, Singh H, Haverich A and Martin U: Scalable expansion of human pluripotent stem cells in suspension culture. Nat Protoc 6: 689-700, 2011.

7. Chen L, Xiao Z, Meng Y, Zhao Y, Han J, Su G, Chen B and Dai J: The enhancement of cancer stem cell properties of MCF-7 cells in 3D collagen scaffolds for modeling of cancer and anti-cancer drugs. Biomaterials 33: 1437-1444, 2012.

8. Su G, Zhao Y, Wei J, Han J, Chen L, Xiao Z, Chen B and Dai J: The effect of forced growth of cells into 3D spheres using low attachment surfaces on the acquisition of stemness properties. Biomaterials 34: 3215-3222, 2013

9. Rao W, Zhao S, Yu J, Lu X, Zynger DL and He X: Enhanced enrichment of prostate cancer stem-like cells with miniaturized 3D culture in liquid core-hydrogel shell microcapsules. Biomaterials 35: 7762-7773, 2014.

10. Palomeras S, Rabionet M, Ferrer I, Sarrats A, Garcia-Romeu ML, Puig T and Ciurana J: Breast cancer stem cell culture and enrichment using poly (ع-caprolactone) scaffolds. Molecules 21: 537, 2016.

11. Moxon SR and Smith AM: Controlling the rheology of gellan gum hydrogels in cell culture conditions. Int J Biol Macromol 84 79-86, 2016 .
12. Pijper A: Methylcellulose and bacterial motility. J Bacteriol 53: 257-269, 1947.

13. García MC, Alfaro MC and Muñoz J: Rheology of sheared gels based on low acyl-gellan gum. Food Sci Technol Int 22: 325-332, 2016.

14. Vilela JA and da Cunha RL: High acyl gellan as an emulsion stabilizer. Carbohydr Polym 139: 115-124, 2016.

15. Livak KJ and Schmittgen TD: Analysis of relative gene expression data using real-time quantitative PCR and the 2(-Delta Delta C(T)) method. Methods 25: 402-408, 2001.

16. Gilbert CA and Ross AH: Cancer stem cells: Cell culture, markers, and targets for new therapies. J Cell Biochem 108: 1031-1038, 2009.

17. Silginer M, Weller M, Ziegler U and Roth P: Integrin inhibition promotes atypical anoikis in glioma cells. Cell Death Dis 5: e1012, 2014.

18. Debnath J and Brugge JS: Modelling glandular epithelial cancers in three-dimensional cultures. Nat Rev Cancer 5: 675-688, 2005.

19. Martinez-Cruzado L, Tornin J, Santos L, Rodriguez A, García-Castro J, Morís F and Rodriguez R: Aldh1 expression and activity increase during tumor evolution in sarcoma cancer stem cell populations. Sci Rep 6: 27878, 2016.

20. Carmody LC, Germain AR, VerPlank L, Nag PP, Muñoz B, Perez JR and Palmer MA: Phenotypic high-throughput screening elucidates target pathway in breast cancer stem cell-like cells. J Biomol Screen 17: 1204-1210, 2012.

21. Prajapati VD, Jani GK, Zala BS and Khutliwala TA: An insight into the emerging exopolysaccharide gellan gum as a novel polymer. Carbohydr Polym 93: 670-678, 2013.

22. Sutherland IW: Novel and established applications of microbial polysaccharides. Trends Biotechnol 16: 41-46, 1998.

23. Ramburrun P, Kumar P, Choonara YE, du Toit LC and Pillay V: Design and characterization of neurodurable gellan-xanthan $\mathrm{pH}-\mathrm{responsive}$ hydrogels for controlled drug delivery. Expert Opin Drug Deliv 14: 291-306, 2017.

24. Yoshida Y, Takahashi K, Okita K, Ichisaka T and Yamanaka S: Hypoxia enhances the generation of induced pluripotent stem cells. Cell Stem Cell 5: 237-241, 2009.

25. Carnero A and Lleonart M: The hypoxic microenvironment: A determinant of cancer stem cell evolution. Bioessays 38 (Suppl 1): S65-S74, 2016.

26. Hongisto V, Jernström S, Fey V, Mpindi JP, Kleivi Sahlberg K, Kallioniemi $\mathrm{O}$ and Perälä M: High-throughput 3D screening reveals differences in drug sensitivities between culture models of JIMT1 breast cancer cells. PLoS One 8: e77232, 2013.

27. Wong GS and Rustgi AK: Matricellular proteins: Priming the tumour microenvironment for cancer development and metastasis. Br J Cancer 108: 755-761, 2013.

28. Plaks V, Kong N and Werb Z: The cancer stem cell niche: How essential is the niche in regulating stemness of tumor cells? Cell Stem Cell 16: 225-238, 2015.

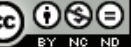

This work is licensed under a Creative Commons Attribution-NonCommercial-NoDerivatives 4.0 International (CC BY-NC-ND 4.0) License. 